\title{
Corrigendum
}

\section{On the use of a discrete form of the Itô formula in the article 'Almost sure asymptotic stability analysis of the $\theta$-Maruyama method applied to a test system with stabilising and destabilising stochastic perturbations'}

\author{
(LMS J. Comput. Math. 15 (2012) 71-83)
}

Gregory Berkolaiko, Evelyn Buckwar, Cónall Kelly and Alexandra Rodkina

\section{ABSTRACT}

In the original article [LMS J. Comput. Math. 15 (2012) 71-83], the authors use a discrete form of the Itô formula, developed by Appleby, Berkolaiko and Rodkina [Stochastics 81 (2009) no. 2, 99-127], to show that the almost sure asymptotic stability of a particular two-dimensional test system is preserved when the discretisation step size is small. In this Corrigendum, we identify an implicit assumption in the original proof of the discrete Itô formula that, left unaddressed, would preclude its application to the test system of interest. We resolve this problem by reproving the relevant part of the discrete Itô formula in such a way that confirms its applicability to our test equation. Thus, we reaffirm the main results and conclusions of the original article.

\section{Introduction}

Consider the linear two-dimensional stochastic differential equation

$$
\begin{aligned}
d\left(\begin{array}{l}
X_{1}(t) \\
X_{2}(t)
\end{array}\right)= & \left(\begin{array}{ll}
\lambda & 0 \\
0 & \lambda
\end{array}\right)\left(\begin{array}{l}
X_{1}(t) \\
X_{2}(t)
\end{array}\right) d t \\
& +\left(\begin{array}{ll}
\sigma & 0 \\
0 & \sigma
\end{array}\right)\left(\begin{array}{l}
X_{1}(t) \\
X_{2}(t)
\end{array}\right) d W_{1}(t)+\left(\begin{array}{cc}
0 & -\varepsilon \\
\varepsilon & 0
\end{array}\right)\left(\begin{array}{l}
X_{1}(t) \\
X_{2}(t)
\end{array}\right) d W_{2}(t), \quad t>0,
\end{aligned}
$$

where $\lambda, \sigma, \varepsilon \in \mathbb{R}$ and $W_{1}, W_{2}$ are independent Wiener processes. Equation (1.1) has an equilibrium solution $\left(X_{1}, X_{2}\right) \equiv(0,0)$ which is globally almost surely asymptotically stable if and only if

$$
2 \lambda-\sigma^{2}+\varepsilon^{2}<0 .
$$

Following discretisation by the $\theta$-Maruyama method, (1.1) becomes the 2-dimensional difference equation

$$
\left(\begin{array}{l}
X_{1, n+1} \\
X_{2, n+1}
\end{array}\right)=\mathcal{A}_{n+1}\left(\begin{array}{l}
X_{1, n} \\
X_{2, n}
\end{array}\right), \quad n \in \mathbb{N}
$$

with random coefficient

$$
\mathcal{A}_{n}=\left(\begin{array}{cc}
\frac{1+(1-\theta) h \lambda}{1-\theta h \lambda}+\frac{\sqrt{h} \sigma \xi_{1, n}}{1-\theta h \lambda} & \frac{-\sqrt{h} \varepsilon \xi_{2, n}}{1-\theta h \lambda} \\
\frac{\sqrt{h} \varepsilon \xi_{2, n}}{1-\theta h \lambda} & \frac{1+(1-\theta) h \lambda}{1-\theta h \lambda}+\frac{\sqrt{h} \sigma \xi_{1, n}}{1-\theta h \lambda}
\end{array}\right), \quad n \in \mathbb{N},
$$

Received 23 January 2013.

2010 Mathematics Subject Classification 60H35 (primary), 34F05, 60H10, 65C30, 93E15 (secondary). 
where $h>0$ is the discretisation step size, $\theta \in[0,1]$, and $\left\{\xi_{1, n}\right\}_{n \in \mathbb{N}}$ and $\left\{\xi_{2, n}\right\}_{n \in \mathbb{N}}$ are independent sequences of standard normal random variables.

In [2], the authors presented a result stating that, for sufficiently small $h$, the almost sure asymptotic stability of the equilibrium solution of (1.3) is also governed by condition (1.2). We reproduce the result here.

TheOREm 1.1. Let $\left\{X_{n}\right\}_{n \in \mathbb{N}}$ be a solution of equation (1.3), and define $\left\{Z_{n}\right\}_{n \in \mathbb{N}}$ such that $Z_{n}=X_{1, n}^{2}+X_{2, n}^{2}$. Then there exists $h_{0}=h_{0}(\lambda, \varepsilon, \theta, \sigma)$ such that for all $h \leqslant h_{0}$.

(i) $\lim _{n \rightarrow \infty} Z_{n}=0$ almost surely if and only if

$$
2 \lambda-\sigma^{2}+\varepsilon^{2}<0
$$

(ii) $\lim _{n \rightarrow \infty} Z_{n}=\infty$ almost surely if and only if

$$
2 \lambda-\sigma^{2}+\varepsilon^{2}>0
$$

However, the proof of Theorem 1.1, as given in [2], relies upon an implicit assumption that is unwarranted in this case. The purpose of this article is to identify and explain the unwarranted assumption, demonstrate that it is unnecessary, and hence confirm that the statement of Theorem 1.1 and the conclusions of [2] are correct.

\section{Identifying the problem}

\subsection{Application of a discrete form of the Itô formula}

The transformation $Z_{n}=X_{1, n}^{2}+X_{2, n}^{2}$ in the statement of Theorem 1.1 has the effect of recasting the 2-dimensional discrete system (1.3) as a 1-dimensional difference equation of the form

$$
Z_{n+1}=Z_{n}\left(1+h F_{h}+\sqrt{h} G_{h} \zeta_{n+1}\right), \quad n \in \mathbb{N} .
$$

By writing (2.1) in the form

$$
Z_{n+1}=Z_{0} \exp \left[\sum_{i=0}^{n} \ln \left(1+h F_{h}+\sqrt{h} G_{h} \zeta_{n+1}\right)\right], \quad n \in \mathbb{N},
$$

the following necessary and sufficient almost sure asymptotic stability condition for equation (2.1) emerges via the strong law of large numbers:

$$
\mathbb{E}\left[\ln \left(1+h F_{h}+\sqrt{h} G_{h} \zeta_{n+1}\right)\right]<0 .
$$

Note that terms of the sequence $\left\{\zeta_{n}\right\}_{n \in \mathbb{N}}$ are independent and identically distributed, and hence the left-hand side of the inequality evaluates to a constant, independent of $n$.

In order to generate from (2.3) a necessary and sufficient condition for almost sure asymptotic stability in terms of the system parameters, a discrete form of the Itô formula, first proved in [1], was applied in [2] to expand the left-hand side of (2.3) for small values of $h$. This required that the sequence $\left\{\zeta_{n}\right\}_{n \in \mathbb{N}}$ satisfy the following assumption.

Assumption 1. We assume that $\left\{\zeta_{n}\right\}$ is a sequence of $\mathcal{F}_{n}$-measurable random variables where

$$
\mathbb{E} \zeta_{n}=0, \quad \mathbb{E} \zeta_{n}^{2}=1, \quad \mathbb{E}\left|\zeta_{n}\right|^{3} \text { are uniformly bounded, }
$$

and each $\zeta_{n}$ has density function $y=p_{n}(x)$ satisfying

$$
x^{3} p_{n}(x) \rightarrow 0 \quad \text { as }|x| \rightarrow \infty \text { uniformly in } n .
$$


The result itself was stated as the following theorem.

Theorem 2.1. Consider $\varphi: \mathbb{R} \rightarrow \mathbb{R}$ such that there exists $\delta>0$ and $\tilde{\varphi}: \mathbb{R} \rightarrow \mathbb{R}$ satisfying:

(i) $\tilde{\varphi} \equiv \varphi$ on $U_{\delta}=[1-\delta, 1+\delta]$;

(ii) $\tilde{\varphi} \in C^{3}(\mathbb{R})$ and $\left|\tilde{\varphi}^{\prime \prime \prime}(x)\right| \leqslant M$ for some $M$ and all $x \in \mathbb{R}$;

(iii) $\int_{\mathbb{R}}|\varphi-\tilde{\varphi}| d x<\infty$.

For each $n$ let $f_{n}$ and $g_{n}$ be $\mathcal{F}_{n}$-measurable uniformly bounded random variables, $\left|f_{n}\right|,\left|g_{n}\right|<K$ and let $\zeta_{n+1}$ be an $\mathcal{F}_{n}$-independent random variable satisfying (2.4) and (2.5) in Assumption 1. Then

$$
\mathbb{E}\left[\varphi\left(1+f_{n} h+g_{n} \sqrt{h} \zeta_{n+1}\right) \mid \mathcal{F}_{n}\right]=\varphi(1)+\varphi^{\prime}(1) f_{n} h+\frac{\varphi^{\prime \prime}(1)}{2} g_{n}^{2} h+h f_{n} O(h)+h g_{n}^{2} O(h),
$$

where the error term $O(h) \rightarrow 0$ as $h \rightarrow 0$, uniformly in $n, f_{n}$ and $g_{n}$.

To understand the motivation for this article, we must review the proof of Theorem 2.1 in outline; details may be found in [1].

Sketch Proof of Theorem 2.1. The proof has two parts. First, it is shown that (2.6) holds for the auxiliary function $\tilde{\varphi}$, by writing the Taylor expansion

$$
\tilde{\varphi}(1+x)=\tilde{\varphi}(1)+\tilde{\varphi}^{\prime}(1)(x)+\frac{\tilde{\varphi}^{\prime \prime}(1)}{2} x^{2}+\frac{\tilde{\varphi}^{\prime \prime \prime}(\vartheta)}{6} x^{3},
$$

with $\vartheta \in(0, x)$, and substituting $x=f_{n} h+g_{n} \sqrt{h} \zeta_{n+1}$. This part of the proof requires that $\tilde{\varphi}^{\prime \prime \prime}(\vartheta)$ be uniformly bounded, and this explains the need for the auxiliary function $\tilde{\varphi}$ : if $\varphi(x)=\log (x)$ then $\varphi^{\prime \prime \prime}(x)$ is not uniformly bounded.

The second part of the proof is devoted to showing that $\tilde{\varphi}$ is a close approximation of $\varphi$, in the sense that (omitting the subscript $n$ )

$$
|\mathbb{E}[\varphi-\tilde{\varphi}]| \leqslant h g^{2} O(h),
$$

and therefore that (2.6) also holds for $\varphi$. Note that in the original proof in [1] it is assumed for brevity that $f$ and $g$ are non-random constants, and the proof therefore examines the non-conditional expectation, with the comment that the conditional version may be treated similarly.

The following estimate is critical: omitting the subscript $n$, set $c_{1}=1+h f$ and $c_{2}=\sqrt{h} g$,

$$
\begin{aligned}
\triangle & :=\mathbb{E}\left[\varphi\left(c_{1}+c_{2} \zeta\right)-\tilde{\varphi}\left(c_{1}+c_{2} \zeta\right)\right] \\
& =\int_{\mathbb{R}}\left(\varphi\left(c_{1}+c_{2} \zeta\right)-\tilde{\varphi}\left(c_{1}+c_{2} \zeta\right)\right) p(\zeta) d \zeta \\
& \leqslant h g^{2} \sup _{r \notin U_{\delta}}\left\{\frac{p(y) y^{3}}{(r-1-h f)^{3}}\right\},
\end{aligned}
$$

where the change of variables $r=c_{1}+c_{2} \zeta$ has been made, and

$$
y=\frac{r-1-h f}{\sqrt{h} g}
$$

satisfies $y \rightarrow \infty$ as $h \rightarrow 0$. We see then, for fixed $r \notin U_{\delta}$, that $(r-1-h f)^{3}$ is bounded away from zero for sufficiently small $h$, and by Assumption 1,

$$
\lim _{y \rightarrow \infty} y^{3} p(y)=0
$$

which gives (2.7) and completes the proof. 


\subsection{Statement of the problem}

Note that in the sketch proof of Theorem 2.1, the statement (2.8) is standing in for, and is implicitly assumed to be equivalent to,

$$
\lim _{h \rightarrow 0}\left[\frac{r-1-h f}{\sqrt{h} g}\right]^{3} p\left(\frac{r-1-h f}{\sqrt{h} g}\right)=0,
$$

for fixed $r$. This is in fact the case if the density $p$ does not itself depend on $h$. However, recall from [2] that terms of the sequence $\left\{\zeta_{n}\right\}_{n \in \mathbb{N}}$ may be expressed explicitly as follows. Defining

$$
\eta_{n}:=\frac{2(1+(1-\theta) \lambda h) \sigma \xi_{1, n}+\sqrt{h} \sigma^{2}\left(\xi_{1, n}^{2}-1\right)+\sqrt{h} \varepsilon^{2}\left(\xi_{2, n}^{2}-1\right)}{(1-\theta \lambda h)^{2}},
$$

we have

$$
\zeta_{n}=\frac{\eta_{n}}{\sqrt{\mathbb{E}\left(\eta_{n}^{2}\right)}} .
$$

So the density function $p_{n}$ of each $\zeta_{n}$ depends very much on $h$ in a way that is difficult to characterise. Therefore, even though it is true [2, Lemma 2.8] that Assumption 1 holds for the density function of each $\zeta_{n}$, the original proof of the discrete form of the Itô formula given by Theorem 2.1 does not work when those densities are $h$-dependent.

\section{Resolving the problem}

The solution is to revisit the second part of the proof of Theorem 2.1 and show that for (2.1), $\tilde{\varphi}$ is a close approximation of $\varphi$ in the sense of (2.7), even though the density function of each $\zeta_{n}$ defined in $(2.10)$ is $h$-dependent.

Recall that Theorem 2.1 was reformulated in [2, Corollary 2.4] as follows before being applied to the left-hand side of (2.3), so that (2.6) takes the form

$$
\mathbb{E}\left[\varphi\left(1+h F_{h}+\sqrt{h} G_{h} \zeta\right) \mid \mathcal{F}_{n}\right]=\varphi(1)+h \varphi^{\prime}(1) f(h)+h \frac{\varphi^{\prime \prime}(1)}{2} g^{2}(h)+h O(h),
$$

where [2, Lemma 2.8]

$$
\begin{aligned}
& F_{h}=f(h)+O(h):=\frac{2 \lambda+\sigma^{2}+\varepsilon^{2}}{(1-\theta \lambda h)^{2}}+O(h), \\
& G_{h}^{2}=g^{2}(h)+O(h):=\frac{4 \sigma^{2}}{(1-\theta \lambda h)^{2}}+O(h) .
\end{aligned}
$$

Note that, since each $\zeta_{n}$ is identically distributed, we omit the subscript in (3.1). For the remainder of the article, let $\zeta$ be a random variable with the same distribution as terms of the sequence $\left\{\zeta_{n}\right\}_{n \in \mathbb{N}}$ as given in (2.10), and let $\eta$ be a random variable with the same distribution as terms of the sequence $\left\{\eta_{n}\right\}_{n \in \mathbb{N}}$, as defined in (2.9). It is then sufficient to prove the following result.

Theorem 3.1. Consider the random variable

$$
U=1+h F_{h}+\sqrt{h} G_{h} \zeta
$$

and

$$
F_{h}=\frac{2 \lambda}{(1-\theta \lambda h)}+\frac{\left(\sigma^{2}+\varepsilon^{2}\right)}{(1-\theta \lambda h)^{2}}+\frac{h \lambda^{2}}{(1-\theta \lambda h)^{2}}, \quad G_{h}=\sqrt{\mathbb{E}\left[\eta^{2}\right]}
$$

Suppose also that $\varphi$ and $\tilde{\varphi}$ satisfy conditions (i) and (ii) of Theorem 2.1, and additionally that

$$
\varphi-\tilde{\varphi} \in L_{s}[-\delta, \delta] \quad \text { for some } s>2 .
$$


Then, for $h$ sufficiently small,

$$
|\mathbb{E}[\varphi(U)-\varphi(\tilde{U})]| \leqslant h g^{2}(h) O(h) .
$$

REMARK 1. Note that the stronger integrability condition for $\varphi-\tilde{\varphi}$ imposed by (3.6) in the statement of Theorem 3.1 is nonetheless satisfied by the specific choices of $\varphi$ and $\tilde{\varphi}$ in the proof of $[\mathbf{2}$, Theorem 3.2].

Proof. We will assume, without loss of generality, that $\sigma, \varepsilon \geqslant 0$. Rewrite (3.4) as

$$
U\left(\xi_{1}, \xi_{2}\right)=1+h F_{h}+2 \sqrt{h} \tilde{\sigma}_{1}(h) \xi_{1}+h \tilde{\sigma}_{2}(h)\left(\xi_{1}^{2}-1\right)+h \tilde{\varepsilon}(h)\left(\xi_{2}^{2}-1\right),
$$

where the functions

$$
\begin{aligned}
\tilde{\sigma}_{1}(h) & :=\frac{\sigma(1+(1-\theta) \lambda h)}{(1-\theta \lambda h)^{2}}, \\
\tilde{\sigma}_{2}(h) & :=\frac{\sigma}{(1-\theta \lambda h)}, \\
\tilde{\varepsilon}(h) & :=\frac{\varepsilon}{(1-\theta \lambda h)},
\end{aligned}
$$

satisfy

$$
\lim _{h \rightarrow 0} \tilde{\sigma}_{1}(h)=\lim _{h \rightarrow 0} \tilde{\sigma}_{2}(h)=\sigma, \quad \lim _{h \rightarrow 0} \frac{\tilde{\sigma}_{1}(h)}{\tilde{\sigma}_{2}(h)}=1 \quad \text { and } \quad \lim _{h \rightarrow 0} \tilde{\varepsilon}(h)=\varepsilon .
$$

Extracting the perfect square gives

$$
\begin{aligned}
U & =U\left(\xi_{1}, \xi_{2}\right) \\
& =h\left(\tilde{\sigma}_{2}(h) \xi_{1}+\frac{\tilde{\sigma}_{1}(h)}{\sqrt{h} \tilde{\sigma}_{2}(h)}\right)^{2}+h \tilde{\varepsilon}^{2}(h) \xi_{2}^{2}+1+h F_{h}-\frac{\tilde{\sigma}_{1}^{2}(h)}{\tilde{\sigma}_{2}^{2}(h)}-h \tilde{\varepsilon}^{2}(h) .
\end{aligned}
$$

Now define

$$
q(h):=1+h F_{h}-\frac{\tilde{\sigma}_{1}^{2}(h)}{\tilde{\sigma}_{2}^{2}(h)}-h \tilde{\varepsilon}^{2}(h)=\left(1-\frac{\tilde{\sigma}_{1}^{2}(h)}{\tilde{\sigma}_{2}^{2}(h)}\right)+h\left[F_{h}-\tilde{\varepsilon}^{2}(h)\right],
$$

where by (3.5) and (3.7) we can see that

$$
\lim _{h \rightarrow 0} q(h)=0 .
$$

It is then possible to express $U$ in the form

$$
U\left(\xi_{1}, \xi_{2}\right)=h\left(\tilde{\sigma}_{2}(h) \xi_{1}+\frac{\tilde{\sigma}_{1}(h)}{\sqrt{h} \tilde{\sigma}_{2}(h)}\right)^{2}+h \tilde{\varepsilon}^{2}(h) \xi_{2}^{2}+q(h) .
$$

Suppose that $|U| \leqslant \delta$. Then $q(h) \leqslant U \leqslant \delta$ and therefore the following inequalities hold:

$$
\begin{aligned}
h \tilde{\varepsilon}^{2}(h) \xi_{2}^{2} & \leqslant \delta-q(h), \\
h\left(\tilde{\sigma}_{2}(h) \xi_{1}+\frac{\tilde{\sigma}_{1}(h)}{\sqrt{h} \tilde{\sigma}_{2}(h)}\right)^{2} & \leqslant \delta-q(h), \\
0 \leqslant U-h \tilde{\varepsilon}^{2}(h) \xi_{2}^{2}-q(h) & \leqslant \delta-q(h) .
\end{aligned}
$$

Solving (3.10) in terms of $\xi_{1}$ we obtain

$$
\xi_{1}=-\frac{\tilde{\sigma}_{1}(h)}{\sqrt{h} \tilde{\sigma}_{2}^{2}(h)} \pm \frac{\sqrt{U-h \tilde{\varepsilon}^{2}(h) \xi_{2}^{2}-q(h)}}{\sqrt{h} \tilde{\sigma}_{2}(h)}=-\frac{\tilde{\sigma}_{1}(h) / \tilde{\sigma}_{2}(h) \pm \sqrt{U-h \tilde{\varepsilon}^{2}(h) \xi_{2}^{2}-q(h)}}{\sqrt{h} \tilde{\sigma}_{2}(h)} .
$$

Applying (3.7) and (3.9) for $h$ sufficiently small, and with $\delta<1 / 4$,

$$
\frac{\tilde{\sigma}_{1}(h)}{\tilde{\sigma}_{2}(h)}-\sqrt{U-h \tilde{\varepsilon}^{2}(h) \xi_{2}^{2}-q(h)} \geqslant \frac{\tilde{\sigma}_{1}(h)}{\tilde{\sigma}_{2}(h)}-\sqrt{\delta-q(h)} \geqslant \frac{1}{2},
$$


and, therefore,

$$
\left|\xi_{1}\right| \geqslant\left|\frac{\tilde{\sigma}_{1}(h) / \tilde{\sigma}_{2}(h)-\sqrt{U-h \tilde{\varepsilon}^{2}(h) \xi_{2}^{2}-q(h)}}{\sqrt{h} \tilde{\sigma}_{2}(h)}\right| \geqslant\left|\frac{\tilde{\sigma}_{1}(h) / \tilde{\sigma}_{2}(h)-\sqrt{\delta-q(h)}}{\sqrt{h} \tilde{\sigma}_{2}(h)}\right| \geqslant \frac{1}{2 \sqrt{h} \tilde{\sigma}_{2}(h)} .
$$

Now we have

$$
\Delta=\mathbb{E}|\varphi(U)-\tilde{\varphi}(U)|=\frac{1}{2 \pi} \iint_{\left|U\left(\xi_{1}, \xi_{2}\right)\right| \leqslant \delta}|\varphi(U)-\tilde{\varphi}(U)| e^{-\frac{1}{2} \xi_{1}^{2}} e^{-\frac{1}{2} \xi_{2}^{2}} d \xi_{1} d \xi_{2} .
$$

We change the variables $\left(\xi_{1}, \xi_{2}\right)$ to $\left(U, \xi_{2}\right)$ in the integral (3.14), selecting the following branch:

$$
\begin{aligned}
& \xi_{1}=-\frac{\tilde{\sigma}_{1}(h)}{\sqrt{h} \tilde{\sigma}_{2}^{2}(h)}+\frac{\sqrt{U-h \tilde{\varepsilon}^{2}(h) \xi_{2}^{2}-q(h)}}{\sqrt{h} \tilde{\sigma}_{2}(h)}, \\
& \xi_{2}=\xi_{2} .
\end{aligned}
$$

The corresponding Jacobian $|J|$ is

$$
|J|=\frac{1}{2 \tilde{\sigma}_{2}(h) \sqrt{h} \sqrt{U-h \tilde{\varepsilon}^{2}(h) \xi_{2}^{2}-q(h)}} .
$$

Denote

$$
u(U):=\sqrt{\frac{U-q(h)}{h \tilde{\varepsilon}^{2}(h)}} .
$$

After changing variables in (3.14), the estimates in (3.11) and (3.13) give

$$
\begin{aligned}
|\Delta| & \leqslant \mathbb{E}|\varphi(U)-\tilde{\varphi}(U)| \\
& =\frac{1}{2 \pi} \int_{-\delta}^{\delta} d U \int_{-u(U)}^{u(U)} \frac{|\varphi(U)-\tilde{\varphi}(U)| e^{-\frac{1}{2}\left(\tilde{\sigma}_{1}(h) / \sqrt{h} \tilde{\sigma}_{2}^{2}(h)-\sqrt{U-h \tilde{\varepsilon}^{2}(h) \xi_{2}^{2}-q(h)} / \sqrt{h} \tilde{\sigma}_{2}(h)\right)^{2}} e^{-\frac{1}{2} \xi_{2}^{2}}}{2 \tilde{\sigma}_{2}(h) \sqrt{h} \sqrt{U-q(h)-h \tilde{\varepsilon}^{2}(h) \xi_{2}^{2}}} d \xi_{2} \\
& \leqslant \frac{1}{2 \pi} \int_{-\delta}^{\delta}|\varphi(U)-\tilde{\varphi}(U)| d U \int_{-u(U)}^{u(U)} \frac{e^{-\frac{1}{2}\left(1 / 2 \sqrt{h} \tilde{\sigma}_{2}(h)\right)^{2}} e^{-\frac{1}{2} \xi_{2}^{2}}}{2 \tilde{\sigma}_{2}(h) \sqrt{h} \sqrt{U-q(h)-h \tilde{\varepsilon}^{2}(h) \xi_{2}^{2}}} d \xi_{2} .
\end{aligned}
$$

Since

$$
e^{-\frac{1}{2} \xi_{2}^{2}} \leqslant 1 \quad \text { and } \quad e^{-1 / x} \leqslant 2 x^{2} \quad \text { for } x>0
$$

we can write

$$
\begin{aligned}
|\Delta| & \leqslant \frac{128 h^{2} \tilde{\sigma}_{2}^{4}(h)}{2 \pi 2 \tilde{\sigma}_{2}(h) \sqrt{h}} \int_{-\delta}^{\delta}|\varphi(U)-\tilde{\varphi}(U)| d U \int_{-u(U)}^{u(U)} \frac{1}{\sqrt{U-q(h)-h \tilde{\varepsilon}^{2}(h) \xi_{2}^{2}}} d \xi_{2} \\
& =\frac{32 h^{3 / 2} \tilde{\sigma}_{2}^{3}(h)}{\pi} \int_{-\delta}^{\delta}|\varphi(U)-\tilde{\varphi}(U)| d U \int_{-u(U)}^{u(U)} \frac{\sqrt{h} \tilde{\varepsilon}(h)}{\sqrt{u^{2}(U)-\xi_{2}^{2}}} d \xi_{2} \\
& =\frac{32 h^{3 / 2} \tilde{\sigma}_{2}^{3}(h) \tilde{\varepsilon}(h)}{\pi} \int_{-\delta}^{\delta}|\varphi(U)-\tilde{\varphi}(U)| d U \int_{-u(U)}^{u(U)} \frac{1}{\sqrt{u^{2}(U)-\xi_{2}^{2}}} d \xi_{2} \\
& =\frac{32 h^{3 / 2} \tilde{\sigma}_{2}^{3}(h) \tilde{\varepsilon}(h)}{\pi} \int_{-\delta}^{\delta}|\varphi(U)-\tilde{\varphi}(U)| d U\left[\left.\frac{1}{u(U)} \arcsin \frac{\xi_{2}}{u(U)}\right|_{-u(U)} ^{u(U)}\right] \\
& =32 h^{3 / 2} \tilde{\sigma}_{2}^{3}(h) \tilde{\varepsilon}(h) \int_{-\delta}^{\delta}|\varphi(U)-\tilde{\varphi}(U)| \frac{1}{\sqrt{U-q(h) / h \sigma_{2}^{2}(h)}} d U \\
& =h^{2} g^{2}(h) \frac{8 \sigma^{2} \varepsilon}{(1-\theta \lambda h)^{3}} \int_{-\delta}^{\delta}|\varphi(U)-\tilde{\varphi}(U)| \frac{1}{\sqrt{U-q(h)}} d U,
\end{aligned}
$$


where $g(h)$ is as defined in (3.3). Applying Hölder's inequality with $s>2$ and $p<2$ such that $1 / s+1 / p=1$ gives

$$
|\Delta| \leqslant h^{2} g^{2}(h) \frac{8 \sigma^{2} \varepsilon}{(1-\theta \lambda h)^{3}}\left(\int_{-\delta}^{\delta}|\varphi(U)-\tilde{\varphi}(U)|^{s} d U\right)^{1 / s}\left(\int_{-\delta}^{\delta} \frac{1}{(U-q(h))^{p / 2}} d U\right)^{1 / p} .
$$

Finally, by applying (3.6) we have

$$
|\Delta| \leqslant h g^{2}(h) O(h),
$$

as required.

REMARK 2. Note from (3.16) that, in order for the statement of Theorem 3.1 to hold, it is sufficient that terms of the sequences $\left\{\xi_{1, n}\right\}_{n \in \mathbb{N}}$ and $\left\{\xi_{2, n}\right\}_{n \in \mathbb{N}}$ be identically distributed with bounded density functions, the tails of which decay faster than $1 / x^{4}$.

\section{Conclusion}

We draw the following conclusions.

(1) The main results and conclusions of [2] are correct.

(2) The proof of the discrete Itô formula developed in [1] must be adapted to the test equation of interest in order to accommodate a perturbation with $h$-dependent density.

(3) There is a general implication for the linear stability analysis of numerical methods for stochastic differential equations: the need to consider more than one test equation in is highlighted in $[\mathbf{3}, \mathbf{4}]$, and this analysis demonstrates that the discrete Itô formula cannot necessarily be applied to different test equations without adapting the proof to the special structure of each.

\section{References}

1. J. A. D. Appleby, G. Berkolaiko and A. Rodkina, 'Non-exponential stability and decay rates in nonlinear stochastic difference equation with unbounded noises', Stochastics 81 (2009) no. 2, 99-127.

2. G. Berkolaiko, E. Buckwar, C. Kelly and A. Rodkina, 'Almost sure asymptotic stability analysis of the $\theta$-Maruyama method applied to a test system with stabilising and destabilising perturbations', LMS J. Comput. Math. 15 (2012) 71-83.

3. E. BuCKWAR and C. KELLY, 'Towards a systematic linear stability analysis of numerical methods for systems of stochastic differential equations', SIAM J. Numer. Anal. 48 (2010) no. 1, 298-321.

4. E. BuCKWAR and C. KELLY, 'Non-normal drift structures and linear stability analysis of numerical methods for systems of stochastic differential equations', Comput. Math. Appl. 64 (2012) no. 7, 2282-2293.

Gregory Berkolaiko

Department of Mathematics

Texas A\&M University

College Station

TX 77843-3368, USA

gregory.berkolaiko@math.tamu.edu

Cónall Kelly

Department of Mathematics

University of the West Indies

Mona, Kingston 7, Jamaica

conall.kelly@uwimona.edu.jm

\author{
Evelyn Buckwar \\ Institute for Stochastics \\ Johannes Kepler University Linz \\ Altenberger Straße 69 \\ 4040 Linz, Austria
}

Evelyn.Buckwar@jku.at

Alexandra Rodkina

Department of Mathematics

University of the West Indies

Mona, Kingston 7, Jamaica

alexandra.rodkina@uwimona.edu.jm 